\title{
Some Multiplicative Inequalities for Inner Products and of the Carlson Type
}

\author{
Sorina Barza, ${ }^{1}$ Lars-Erik Persson, $^{2}$ and Emil C. Popa ${ }^{3}$ \\ ${ }^{1}$ Department of Mathematics, Karlstad University, 65188 Karlstad, Sweden \\ ${ }^{2}$ Department of Mathematics, Lulea University of Technology, 97187 Luleå, Sweden \\ ${ }^{3}$ Department of Mathematics, "Lucian Blaga" University of Sibiu, 550024 Sibiu, Romania
}

Correspondence should be addressed to Sorina Barza, sorina.barza@kau.se

Received 26 September 2007; Accepted 17 January 2008

Recommended by Wing-Sum Cheung

We prove a multiplicative inequality for inner products, which enables us to deduce improvements of inequalities of the Carlson type for complex functions and sequences, and also other known inequalities.

Copyright $(0) 2008$ Sorina Barza et al. This is an open access article distributed under the Creative Commons Attribution License, which permits unrestricted use, distribution, and reproduction in any medium, provided the original work is properly cited.

\section{Introduction}

Let $\left(a_{n}\right)_{n=1}^{\infty}$ be a nonzero sequence of nonnegative numbers and let $f$ be a measurable function on $[0, \infty)$. In 1934, Carlson [1] proved that the inequalities

$$
\begin{gathered}
\left(\sum_{n=1}^{\infty} a_{n}\right)^{4}<\pi^{2}\left(\sum_{n=1}^{\infty} a_{n}^{2}\right)\left(\sum_{n=1}^{\infty} n^{2} a_{n}^{2}\right) \\
\left(\int_{0}^{\infty} f(x) d x\right)^{4} \leq \pi^{2}\left(\int_{0}^{\infty} f^{2}(x) d x\right)\left(\int_{0}^{\infty} x^{2} f^{2}(x) d x\right)
\end{gathered}
$$

hold and $C=\pi^{2}$ is the best constant in both cases. Several generalizations and applications in different branches of mathematics were given during the years. For a complete survey of the results and applications concerning the above inequalities and also historical remarks, see the book [2]. In particular, some multiplicative inequalities of the type

$$
\left(\int_{0}^{\infty} f(x) d x\right)^{4} \leq C\left(\int_{0}^{\infty} w_{1}^{2}(x) f^{2}(x) d x\right)\left(\int_{0}^{\infty} w_{2}^{2}(x) f^{2}(x) d x\right)
$$


are known for special weight functions $w_{1}$ and $w_{2}$, where usually $w_{1}$ and $w_{2}$ are power functions or homogeneous. In this paper, we prove a refined version of (1.3) for a fairly general class of weight functions (see Corollary 3.2). In particular, this inequality shows that (1.2) holds with the constant $\pi^{2}$ for many infinite weights beside the classical ones $w_{1}(x)=1$ and $w_{2}(x)=x$. Our method of proof is different from the other proofs (e.g., those by Larsson et al. presented in the book [2]) and the basic idea is to first prove a more general multiplicative inequality for inner products (see Theorem 2.3). Some similar improvements and complements of (1.1) are also included.

The paper is organized as follows: in Section 2 we prove our general multiplicative inequality for inner products. In Section 3 we deduce an integral inequality of the Carlson type for general measure spaces and prove some corollaries for the Lebesgue measure and the counting measure, which are improvements of inequalities (1.2) and (1.1). Section 4 is devoted to an inequality for an inner product defined on a space of square matrices, which is a generalization of known discrete inequalities.

\section{A multiplicative inequality for inner products}

Let $(X,+, \cdot)$ be a vector space over a scalar field $\mathbb{R}$ or $\mathbb{C}$ and let $F: X \times X \rightarrow \mathbb{C}$ be an inner product on $X$. First, we formulate the following Lemma.

Lemma 2.1. Let $x, y \in X$ be such that $x, y \neq 0$. Then there exists $\lambda \in \mathbb{C}, \lambda \neq 0$ such that $\operatorname{Re}((\lambda / \bar{\lambda}) F(x, y))=0$ and $|\lambda|^{2}=\sqrt{F(y, y) / F(x, x)}$.

Proof. Let $x, y \in X$ be such that $x, y \neq 0$. Then $F(x, x), F(y, y)>0$, and $F(x, y)=|F(x, y)| e^{i \varphi}$ for some $\varphi \in[0,2 \pi)$. If $F(x, y)=0$, then $\varphi$ is arbitrary. Set $\lambda=\sqrt[4]{F(y, y) / F(x, x)} e^{i( \pm \pi / 4-\varphi / 2)}$. Then $|\lambda|^{2}=\sqrt{F(y, y) / F(x, x)}>0, \lambda / \bar{\lambda}=e^{i( \pm \pi / 2-\varphi)}$, and $(\lambda / \bar{\lambda}) F(x, y)=|F(x, y)| e^{ \pm i(\pi / 2)}$, so $\operatorname{Re}((\lambda / \bar{\lambda}) F(x, y))=0$ and the proof is completed.

Remark 2.2. It is observed that the same result can be achieved also with $\lambda=$ $\sqrt[4]{F(y, y) / F(x, x)} e^{i( \pm 3 \pi / 4-\varphi / 2)}$. Thus, for $F(x, y) \in \mathbb{R}$, we have $\lambda=p \pm p i$, where $p^{2}=$ $(1 / 2) \sqrt{F(y, y) / F(x, x)}$.

Our multiplicative inequality of the Carlson type reads as follows.

Theorem 2.3. Let $x, y, v \in X$ be such that $x, y \neq 0$ and let $\lambda$ be any of the numbers satisfying the conditions of Lemma 2.1. Then the inequality

$$
\left|F\left(\lambda x+\frac{1}{\lambda} y, v\right)\right|^{4} \leq 4 F(x, x) F(y, y) F^{2}(v, v)
$$

holds.

Proof. By using Schwarz inequality, we find that

$$
\begin{aligned}
\left|F\left(\lambda x+\frac{1}{\lambda} y, v\right)\right|^{2} & \leq F\left(\lambda x+\frac{1}{\lambda} y, \lambda x+\frac{1}{\lambda} y\right) F(v, v) \\
& =\left(|\lambda|^{2} F(x, x)+\frac{1}{|\lambda|^{2}} F(y, y)+2 \operatorname{Re}\left(\frac{\lambda}{\bar{\lambda}} F(x, y)\right)\right) F(v, v) .
\end{aligned}
$$


By, now, applying Lemma 2.1 and our assumptions on $\lambda$, we find that the right-hand side of (2.2) is equal to $2 \sqrt{F(x, x)} \sqrt{F(y, y)} F(v, v)$ and (2.1) follows.

\section{Inequalities of the Carlson type}

Let $(\Omega, d \mu)$ be a measure space and let $f, g: \Omega \rightarrow \mathbb{C}$ be measurable functions. We define

$$
F(f, g)=\int_{\Omega} f(t) \overline{g(t)} d \mu
$$

which is a standard inner product on $L^{2}(\Omega, d \mu)$. Now, we state and prove the following new Carlson-type inequality.

Theorem 3.1. Let $f: \Omega \rightarrow \mathbb{C}$ and $w_{1}, w_{2}: \Omega \rightarrow \mathbb{R}$ be such that $w_{1} f, w_{2} f \neq 0$ a.e., $f \in L_{w_{1}^{2}}^{2}(\Omega, d \mu) \cap$ $L_{w_{2}^{2}}^{2}(\Omega, d \mu)$ and $|\lambda|^{2} w_{1}^{2}+\left(1 /|\lambda|^{2}\right) w_{2}^{2}>0$, where

$$
\lambda=p \pm p i, \quad p^{2}=(1 / 2) \sqrt{\frac{\int_{\Omega} w_{2}^{2}(x) f(x) d \mu}{\int_{\Omega} w_{1}^{2}(x) f(x) d \mu}}
$$

Then

$$
\left|\int_{\Omega} f(x) d \mu\right|^{4} \leq 4\left(\int_{\Omega} \frac{d \mu}{\left|\lambda w_{1}(x)+(1 / \lambda) w_{2}(x)\right|^{2}}\right)^{2}\left(\int_{\Omega} w_{1}^{2}(x)|f(x)|^{2} d \mu\right)\left(\int_{\Omega} w_{2}^{2}(x)|f(x)|^{2} d \mu\right) .
$$

Proof. In the inner product defined in (3.1) we substitute $f w_{1}$ and $f w_{2}$ for respectively $f$ and $g$ and observe that in this case the number $F\left(f w_{1}, f w_{2}\right)=\int_{\Omega} w_{1}(x) w_{2}(x)|f(x)|^{2} d \mu$ is real. Since $\operatorname{Im} F\left(w_{1} f, w_{2} f\right)=0$, by arguing as in the proof of Lemma 2.1 we find that $\lambda=p \pm p i$, where $p^{2}=(1 / 2) \sqrt{\int_{\Omega} w_{2}^{2}(x)|f(x)|^{2} d \mu / \int_{\Omega} w_{1}^{2}(x)|f(x)|^{2} d \mu}$ fulfills the conditions of Theorem 2.3 , so the inequality (3.3) follows from the inequality (2.1) by taking $v(x)=1 /\left(\lambda w_{1}(x)+(1 / \lambda) w_{2}(x)\right)$. The proof is complete.

The following corollary of the above theorem is an improvement of [3, Theorem 2.1].

Corollary 3.2. For $a \in \mathbb{R}$, let $f:[a, \infty) \rightarrow \mathbb{C}$ be an integrable function and let $w_{1}, w_{2}:[a, \infty) \rightarrow \mathbb{R}_{+}$ be two continuously differentiable functions such that $0<m=\inf _{x>a}\left(w_{2}^{\prime}{ }_{2}(x) w_{1}(x)-w_{2}(x) w_{1}^{\prime}(x)\right)<$ $\infty$ and $\lim _{x \rightarrow \infty} w_{2}(x) / w_{1}(x)=\infty$. Then

$$
\begin{aligned}
\left|\int_{a}^{\infty} f(x) d x\right|^{4} \leq & \left(\frac{\pi}{m}-\frac{2}{m} \arctan \frac{w_{2}(a) \sqrt{\int_{a}^{\infty} w_{1}^{2}(x)|f(x)|^{2} d x}}{w_{1}(a) \sqrt{\int_{a}^{\infty} w_{2}^{2}(x)|f(x)|^{2} d x}}\right)^{2} \\
& \times\left(\int_{a}^{\infty} w_{1}^{2}(x)|f(x)|^{2} d x\right)\left(\int_{a}^{\infty} w_{2}^{2}(x)|f(x)|^{2} d x\right) .
\end{aligned}
$$


Remark 3.3. For the special case when $a=0, w_{2}(0)=0$, and $m=1$, the inequality (3.4) reads

$$
\left|\int_{0}^{\infty} f(x) d x\right|^{4} \leq \pi^{2}\left(\int_{0}^{\infty} w_{1}^{2}(x)|f(x)|^{2} d x\right)\left(\int_{0}^{\infty} w_{2}^{2}(x)|f(x)|^{2} d x\right)
$$

and also this generalization of (1.2) seems to be new (see [2] and the references given there).

Proof. Let $\Omega=[a, \infty)$ and $\mu$ be the Lebesgue measure in inequality (3.3).

Easy calculations show that our assumptions imply that

$$
\begin{gathered}
\left|\lambda^{2}+\frac{w_{2}(x)}{w_{1}(x)}\right|^{2}=|\lambda|^{4}+\frac{w_{2}^{2}(x)}{w_{1}^{2}(x)}, \\
\frac{1}{m}\left(\frac{w_{2}(x)}{w_{1}(x)}\right)^{\prime} \geq \frac{1}{w_{1}^{2}(x)} .
\end{gathered}
$$

Hence, we get that

$$
\begin{aligned}
\int_{a}^{\infty} \frac{d x}{\left|\lambda w_{1}(x)+(1 / \lambda) w_{2}(x)\right|^{2}} & =\int_{a}^{\infty} \frac{|\lambda|^{2} / w_{1}^{2}(x)}{\left|\lambda^{2}+\left(w_{2}(x) / w_{1}(x)\right)\right|^{2}} d x \\
& =\int_{a}^{\infty} \frac{1 /|\lambda|^{2} w_{1}^{2}(x)}{1+\left(w_{2}(x) /|\lambda|^{2} w_{1}(x)\right)^{2}} d x \\
& \leq \frac{1}{m} \int_{a}^{\infty} \frac{\left(w_{2}(x) /|\lambda|^{2} w_{1}(x)\right)^{\prime}}{1+\left(w_{2}(x) /|\lambda|^{2} w_{1}(x)\right)^{2}} d x \\
& =\left[\frac{1}{m} \arctan \frac{w_{2}(x)}{|\lambda|^{2} w_{1}(x)}\right]_{a}^{\infty} \\
& =\frac{\pi}{2 m}-\frac{1}{m} \arctan \frac{w_{2}(a) \sqrt{\int_{a}^{\infty} w_{1}^{2}(x)|f(x)|^{2} d x}}{w_{1}(a) \sqrt{\int_{a}^{\infty} w_{2}^{2}(x)|f(x)|^{2} d x}}
\end{aligned}
$$

and, by using Theorem 3.1, the proof follows.

Remark 3.4. As in [4], we can prove that the condition $\lim _{x \rightarrow \infty} w_{2}(x) / w_{1}(x)=\infty$ cannot be weakened, it is also necessary for our inequality.

Let, now, $\Omega=\mathbb{N}$ and

$$
X=l_{2}(w)=\left\{a=\left(a_{n}\right)_{n=1}^{\infty}: a_{n} \in \mathbb{C}, \sum_{n=1}^{\infty}\left|a_{n}\right|^{2} w_{n}<\infty\right\},
$$

where $w=\left(w_{n}\right)_{n=1}^{\infty}$ is a nontrivial sequence of nonnegative real numbers. Then the functional

$$
F(a, b)=\sum_{n=1}^{\infty} a_{n} \overline{b_{n}} w_{n}
$$

is obviously an inner product on $l_{2}(w)$. Now, we are able to state the following result which is a direct consequence of Theorem 3.1. 
Corollary 3.5. Let $\left(\alpha_{n}\right)_{n=1}^{\infty},\left(\beta_{n}\right)_{n=1}^{\infty}$ be two nontrivial sequences of complex numbers. Then

$$
\left|\sum_{n=1}^{\infty} a_{n} w_{n}\right|^{4} \leq 4\left(\sum_{n=1}^{\infty} \frac{w_{n}}{\left|\lambda \alpha_{n}+(1 / \lambda) \beta_{n}\right|^{2}}\right)^{2}\left(\sum_{n=1}^{\infty}|\alpha|_{n}^{2}\left|a_{n}\right|^{2} w_{n}\right)\left(\sum_{n=1}^{\infty}|\beta|_{n}^{2}\left|a_{n}\right|^{2} w_{n}\right)
$$

for any sequence $\left(a_{n}\right)_{n=1}^{\infty} \subset \mathbb{C}$ of complex numbers, where

$$
\lambda=p \pm p i, \quad p^{2}=(1 / 2) \sqrt{\frac{\sum_{n=1}^{\infty} \alpha_{n}^{2}\left|a_{n}\right|^{2} w_{n}}{\sum_{n=1}^{\infty} \beta_{n}^{2}\left|a_{n}\right|^{2} w_{n}}}
$$

Proof. The proof follows by using Theorem 3.1 with $\Omega=\mathbb{N}$ and $d \mu=\sum_{i=1}^{\infty} w_{i} \delta_{i}$.

Finally, we also include another discrete Carlson-type inequality for complex sequences, which in particular generalizes [3, Theorem 3.1].

Corollary 3.6. Let $\left(a_{n}\right)_{n=1}^{\infty}$ be a sequence of complex numbers and let $\alpha(x), \beta(x)$ be two positive continuously differentiable functions on $[0, \infty)$ such that $0<m=\inf _{x>0}\left(\beta^{\prime}(x) \alpha(x)-\beta(x) \alpha^{\prime}(x)\right)<\infty$. Suppose also that $\alpha(x)$ is increasing, $\lim _{x \rightarrow \infty} \beta(x) / \alpha(x)=\infty$ and $\lim _{x \rightarrow 0} \beta(x) / \alpha(x)=0$. Then the following inequality holds:

$$
\begin{aligned}
\left|\sum_{n=1}^{\infty} a_{n}\right|^{4} \leq & \left(\frac{\pi}{m}-2|\lambda|^{2} \sum_{n=1}^{\infty} \frac{|\lambda|^{4} \alpha\left(c_{n}\right) \alpha^{\prime}\left(c_{n}\right)+\beta\left(c_{n}\right) \beta^{\prime}\left(c_{n}\right)}{\left(|\lambda|^{4} \alpha^{2}\left(c_{n}\right)+\beta^{2}\left(c_{n}\right)\right)^{2}}\right)^{2} \\
& \times\left(\sum_{n=1}^{\infty}\left|a_{n}\right|^{2} \alpha^{2}(n)\right)\left(\sum_{n=1}^{\infty}\left|a_{n}\right|^{2} \beta^{2}(n)\right)
\end{aligned}
$$

for some numbers $c_{n} \in(n-1, n), n \in \mathbb{N}$, where $\lambda \in \mathbb{C}$ is such that

$$
|\lambda|^{2}=\sqrt{\frac{\sum_{n=1}^{\infty} \beta^{2}(n)\left|a_{n}\right|^{2}}{\sum_{n=1}^{\infty} \alpha^{2}(n)\left|a_{n}\right|^{2}}}
$$

Remark 3.7. For the special case when $m=1$ (i.e., when $\inf _{x>0} \beta^{\prime}(x) \alpha(x)=1$ ), the inequality

$$
\left|\sum_{n=1}^{\infty} a_{n}\right|^{4} \leq \pi^{2}\left(\sum_{n=1}^{\infty}\left|a_{n}\right|^{2} \alpha^{2}(n)\right)\left(\sum_{n=1}^{\infty}\left|a_{n}\right|^{2} \beta^{2}(n)\right)
$$

and also the generalization of inequality (1.1) in this simple form seem to be new.

Proof. Let $w_{n}=1$ for any $n \in \mathbb{N}, \alpha_{n}=\alpha(n)$ and $\beta_{n}=\beta(n)$ in Corollary 3.5. We have also

$$
\sum_{n=1}^{\infty} \frac{1}{\left|\lambda \alpha_{n}+(1 / \lambda) \beta_{n}\right|^{2}}=\sum_{n=1}^{\infty} \frac{|\lambda|^{2} / \alpha_{n}^{2}}{|\lambda|^{4}+\beta_{n}^{2} / \alpha_{n}^{2}}
$$


Fix $N \in \mathbb{N}$. Since the function $\varphi(x)=\left(|\lambda|^{2} / \alpha(x)^{2}\right) /\left(|\lambda|^{4}+\beta^{2}(x) / \alpha^{2}(x)\right)$ is decreasing, we have that

$$
\begin{aligned}
\sum_{n=1}^{\infty} \frac{|\lambda|^{2} / \alpha_{n}^{2}}{|\lambda|^{4}+\beta_{n}^{2} / \alpha_{n}^{2}} & <\int_{N}^{\infty} \varphi(x) d x+\sum_{n=1}^{N} \varphi(n) \\
& =\int_{0}^{\infty} \varphi(x) d x-\left(\int_{0}^{N} \varphi(x) d x-\sum_{n=1}^{N} \varphi(n)\right) \\
& \leq \frac{1}{m}\left[\arctan \frac{\beta(x)}{|\lambda|^{2} \alpha(x)}\right]_{0}^{\infty}-\left(\sum_{n=1}^{N} \int_{n-1}^{n}(\varphi(x)-\varphi(n)) d x\right) \\
& \leq \frac{\pi}{2 m}+\frac{1}{2} \sum_{n=1}^{N} \varphi^{\prime}\left(c_{n}\right),
\end{aligned}
$$

where $c_{n}$ are points between $n-1$ and $n$ from the Lagrange mean-value theorem. By differentiating, we find that

$$
\sum_{n=1}^{\infty} \frac{1}{\left|\lambda \alpha_{n}+(1 / \lambda) \beta_{n}\right|^{2}} \leq \frac{\pi}{2 m}-|\lambda|^{2} \sum_{n=1}^{N} \frac{|\lambda|^{4} \alpha\left(c_{n}\right) \alpha^{\prime}\left(c_{n}\right)+\beta\left(c_{n}\right) \beta^{\prime}\left(c_{n}\right)}{\left(|\lambda|^{4} \alpha^{2}\left(c_{n}\right)+\beta^{2}\left(c_{n}\right)\right)^{2}}
$$

where

$$
|\lambda|^{2}=\sqrt{\frac{\sum \beta^{2}(n)\left|a_{n}\right|^{2}}{\sum \alpha^{2}(n)\left|a_{n}\right|^{2}}}
$$

which, by letting $N \rightarrow \infty$ and using (3.10), implies (3.12), and the proof is complete.

\section{Multiplicative inequalities for matrices}

Let $n \in \mathbb{N}$ and $X$ be the vector space of $n \times n$ complex matrices. We denote by $\operatorname{tr}(A)$ the trace of the matrix $A$ and by $A^{*}$ the Hermitian adjoint of $A$, that is, $A^{*}=\overline{A^{t}}$. It is well known that $(A B)^{*}=B^{*} A^{*}$ and $\left(A^{*}\right)^{*}=A$; see, for example, [5]. Moreover, a matrix $A$ is called unitary if $A A^{*}=I_{n}$, where $I_{n}$ is the unity matrix (see, e.g., [5]). We define

$$
F(A, B)=\operatorname{tr}\left(B^{*} A\right)
$$

which is an inner product on $X$ since $F(A+B, C)=\operatorname{tr}\left(C^{*}(A+B)\right)=\operatorname{tr}\left(C^{*} A\right)+\operatorname{tr}\left(C^{*} B\right)=$ $F(A, C)+F(B, C)$. We have also that

$$
F(A, B)=\operatorname{tr}\left(B^{*} A\right)=\sum_{j=1}^{n} \sum_{k=1}^{n} a_{k j} \overline{b_{k j}}=\overline{\sum_{j=1}^{n} \sum_{k=1}^{n} \overline{a_{k j}} b_{k j}}=\overline{\operatorname{tr}\left(A^{*} B\right)} .
$$

The other properties of the inner product are obvious. The inequality (2.1) becomes in this case

$$
\left|\operatorname{tr}\left(C^{*}\left(\lambda A+\frac{1}{\lambda} B\right)\right)\right|^{4} \leq 4 \operatorname{tr}^{2}\left(C^{*} C\right) \operatorname{tr}\left(A^{*} A\right) \operatorname{tr}\left(B^{*} B\right),
$$

where $\lambda$ is one of the complex numbers satisfying the conditions of Lemma 2.1. We can now formulate the following result. 
Proposition 4.1. Let $P, W_{1}, W_{2}$ be $n \times n$ complex matrices such that $W_{1} P, W_{2} P \neq 0$. Then

$$
|\operatorname{tr}(P)|^{4} \leq 4 \operatorname{tr}^{2}\left(\left(\lambda W_{1}+\frac{1}{\lambda} W_{2}\right)^{-1}\left(\left(\lambda W_{1}+\frac{1}{\lambda} W_{2}\right)^{-1}\right)^{*}\right) \operatorname{tr}\left(P^{*} W_{1}^{*} W_{1} P\right) \operatorname{tr}\left(P^{*} W_{2}^{*} W_{2} P\right),
$$

where $\lambda \in \mathbb{C}$ is the parameter defined in Lemma 2.1 (related to the matrices $W_{1} P$ and $W_{2} P$ ), such that $\lambda W_{1}+(1 / \lambda) W_{2}$ is a regular matrix.

Proof. If we substitute $A=W_{1} P, B=W_{2} P, C=\left(\left(\lambda W_{1}+(1 / \lambda) W_{2}\right)^{-1}\right)^{*}$ in (4.3), we get inequality (4.4).

Remark 4.2. If $W_{1}=W_{2}=(\sqrt{2} / 2) W$ where $W$ is a unitary matrix, then $\lambda=\sqrt{2} / 2+(\sqrt{2} / 2) i$ satisfies the conditions of Lemma 2.1. Since $\lambda+1 / \lambda=\sqrt{2}$, the inequality (4.4) becomes

$$
|\operatorname{tr}(P)|^{2} \leq n \operatorname{tr}\left(P^{*} P\right)
$$

and it holds for any $n \times n$ complex matrix $P$. In particular, for diagonal matrices $P=$ $\operatorname{diag}\left(a_{1}, \ldots, a_{n}\right)$, we get the well-known inequality

$$
\left|\sum_{k=1}^{n} a_{k}\right|^{2} \leq n \sum_{k=1}^{n}\left|a_{k}\right|^{2}
$$

for $a_{k} \in \mathbb{C}, k=1, \ldots, n$.

\section{Acknowledgments}

The authors thank the referees for some valuable comments and remarks. They also thank one of the referees for the generosity to even suggest simplifications of one of the proofs.

\section{References}

[1] F. Carlson, "Une inégalité," Arkiv för Matematik, Astronomi och Fysik B, vol. 25, no. 1, pp. 1-5, 1934.

[2] L. Larsson, L. Maligranda, J. Pečarić, and L.-E. Persson, Multiplicative Inequalities of Carlson Type and Interpolation, World Scientific, Hackensack, NJ, USA, 2006.

[3] S. Barza and E. C. Popa, "Weighted multiplicative integral inequalities," Journal of Inequalities in Pure and Applied Mathematics, vol. 7, no. 5, article 169, p. 6, 2006.

[4] L. Larsson, "A new Carlson type inequality," Mathematical Inequalities \& Applications, vol. 6, no. 1, pp. $55-79,2003$.

[5] R. A. Horn and C. R. Johnson, Matrix Analysis, Cambridge University Press, Cambridge, UK, 1985. 